(Aus dem physiologischen Institut der Universität Königsberg i. Pr.)

\title{
Weiteres über die Wirkung starker Ströme auf den Querschnitt der Nerven und Muskeln.
}

\author{
Von
}

\section{Hermann.}

Vor Kurzem habe ich in diesem Archiv Bd. 67 S. 240 eine merkwürdige Erscheinung mitgetheilt, welche man an in Wasser versenkten, longitudinal durchströmten Nerven- und Muskelfasern beobachtet ${ }^{1}$ ). Bei weiterer Verfolgung dieses Phänomens habe ich dadurch Fortschritte machen können, dass es mir gelang, die vorläufig als „Einrollung" bezeichnete störende Gerinnungserscheinung, welche die Haupterscheinung meist bald unterbrach, möglichst zu vermeiden. Da die Einrollung, wie früher berichtet, anscheinend von einem elektrolytischen Product herrührt, das von den Metallrändern her in der Flüssigkeit sich ausbreitet, habe ich die Entfernung zwischen Präparat und Metallrändern gegen früher beträchtlich vergrössert, indem ich die durehströmte Flüssigkeitsstrecke verlängerte. Zugleich wurde, um grosse Stromdichte zu gewinnen, die Breite der Strecke vermindert, indem als Deckglas nicht ein gewöhnliches quadratisches, sondern ein schmales rechteckiges Glasstück verwendet wurde. Um bei dem jetzt sehr vergrösserten Widerstande hinreichende Stromstärken zu gewinnen, wurden sehr hohe Potentialdifferenzen, bis zu 140 Volt, herangezogen.

1) In dieser Arbeit habe ich (S. 246) einige ältere Beobachtungen über Austritt von Nervenmark in Wasser, Säuren u. dgl. angeführt. Nachträglich möchte ich hier noch eine Mittheilung von H. Vi erordt erwähnen (Arch. d. Heilkunde Bd. 18 S. 179, 1876), welcher an eingetrockneten Nerven beim Zerfasern in destillirtem Wasser einen fächerförmigen Austritt des Markes beobachtete; mit dieser, offenbar im Vergleich zu dem galvanischen Austritt geringfügigen Erscheinung (als Austrittslänge wird 3-4 Faserbreiten angegeben) sind die von mir bei blosser Wasserwirkung gesehenen und a. a. 0. erwähnten Austritte anscheinend identisch. 
Das Verfahren ist also nunmehr folgendes:

Auf eine Glasplatte von $180 \mathrm{~mm}$ Länge und $45 \mathrm{~mm}$ Breite (für das grosse Z eiss'sche Stativ) sind zwei sehr dünne (ca. $0,01 \mathrm{~mm}$ ) Stanniolblätter von $30 \mathrm{~mm}$ Breite geklebt, dergestalt, dass dieselben zwischen sich einen Raum von $23 \mathrm{~mm}$ Länge freilassen, und beide Schmalseiten der Glasplatte erreichen, um die Reitklemmen aufzunehmen. Als Deckglas dient ein etwa $30 \mathrm{~mm}$ langes und $5 \mathrm{~mm}$ breites Stück dünnen Objectträgerglases. Das kurze Stück Nerv oder Mnskel (3-5 mm lang) kommt longitudinal in die Mitte zu liegen. Der capillare Raum unter dem Deckglase, dessen Höhe, in Anbetracht der Dicke. des Stanniols und seiner Klebschicht, auf mindestens $0,03 \mathrm{~mm}$ zu veranschlagen ist, und durch dickere Präparate entsprechend grösser wird, muss ganz, ohne jede Luftblase, von Wasser erfüllt sein, und besonders ist darauf zu achten, dass das Wasser beiderseits den Stanniol erreicht und den Raum zwischen Stanniol und Deckglas mit erfüllt. Als Kette diente in der Regel der ganze Accumulator des Instituts, $d$. b. 36 Elemente, zusammen etwa 70 Volt. Oft wurden noch zwei Tauchbatterien, von 20 , resp. 18 Elementen mit in den Kreis aufgenommen, d. h. noch etwa $35-70$ Volt.

Trotz dieser hohen elektromotorischen Kräfte ist bei der Länge und dem geringen Querschnitt (ca. 0,15 qm) der Wasserstrecke die Stromstärke so gering, dass man kaum etwas von Gasentwicklung am Stanniol sieht. Bei besser leitenden Flüssigkeiten, namentlich physiologischer Kochsalzlösung (s. unten), tritt dagegen beträchtliche Gasentwicklung ein, welche durch Aufhebung des Contactes sehr störend wirken kann, wenn man auch hier grosse Spannungen verwendet.

Schon in der ersten Mittheilung babe ich angeführt, dass der anodische Inhaltsaustritt des Nerven in physiologischer Kochsalzlösung ausbleibt, was sich seitdem immer wieder bestätigt hat; dagegen ist er in destillirtem Wasser und in verdünntem Alkohol grossartig. Der Grund liegt, wie schon bemerkt, darin, dass die $0,6 \%$ ige Kochsalzlösung eine zu gut leitende Nebensehliessung zum schlecht leitenden Nerven bildet. Es kommt für die Erscheinung eben nicht auf die Stromdichte in der Flüssigkeit, sondern auf diejenige im Nerven an. An diesem Umstande scheitert auch der Versuch, diejenige Stromdichte direct durch Intensitäts- und Querschnittsmessung zu ermitteln, welche für das Zustandekommen der Erscheinung nöthig ist. Um diese Aufgabe trotzdem einigermaassen zu lösen, suchte ich nach Flüssigkeiten, deren Leitungsvermögen zwischen dem des Wassers und dem der 0,6\%igen Kochsalzlösung liegt und also derjenigen des Nerven näher kommt, so dass man annehmen darf, dass die Stromdichte im Nerven nicht sehr stark von der der umgebenden Flüssigkeit verschieden ist. So gelangte ich zur An- 
Weiteres üb. d. Wirk. starker Ströme a. d. Querschnitt d. Nerven u. Muskeln. 515

wendung einer Kochsalzlösung von $0,1 \%$, in welcher der Austritt sehr schön, und mit geringeren Spannungen als in destillirtem Wasser zu Stande kommt.

Für diese Lösung lässt sich nun die den Versuchsbedingungen entsprechende Stromdichte leicht in absolutem Maasse annähernd feststellen. Ich erinnere daran, dass ich als Einheit für Stromdichten die Grössen $d$ und $\delta$ vorgeschlagen habe ${ }^{1}$ ), welehe seitdem schon von mehreren Autoren acceptirt worden sind. $\Delta$ bedeutet eine Stromstärke von 1 Ampère auf $1 \mathrm{qm}$ Querschnitt, und $\delta$ den millionten Theil dieser Dichte, d. h. 0,001 Milli-Ampère pro qm. In unserem Falle, wo gegen den Widerstand der durchströmten Flüssigkeitssäule alle übrigen Widerstände des Kreises verschwinden, ist die Dichte unabbängig vom Quersehnitt $q$ der Flüssigkeit und nur abbängig von der Länge $l$ ihrer Säule, von ihrem Leitungsvermögen $\lambda$, und von der elektromotorischen Kraft $E$; es ist nämlich

$$
\begin{aligned}
& \text { der Widerstand } w=\frac{l}{\lambda q}, \\
& \text { die Intensität } i=\frac{E}{w}=\frac{\varepsilon \lambda q}{l}, \\
& \text { also die Dichte } \frac{i}{q}=\frac{E \lambda}{l} .
\end{aligned}
$$

Drückt man $l$ in Metern aus (in unserm Falle also $l=0,023$ ), $\lambda$ durch die Verhältnisszahl des Leitungsvermögens zu demjenigen des Quecksilbers, $E$ in Volt, so gibt der angeführte Ausdruck für die Dichte dieselbe in unserem Maasse $\Delta$ an, wenn man den erhaltenen Werth noch durch 1,06 (das Verhältniss zwischen $0 \mathrm{hm}$ und Siemens'scher Quecksilbereinheit) dividirt, was übrigens für die erreichbare Genauigkeit kaum in Betracht kommt. Das Millionfache der erhaltenen Werthe gibt also die Dichte in $\delta$-Einheiten an.

Der ungefähre Betrag von $\lambda$ ist nun bei Zimmertemperatur:

für möglichst reines destillirtes Wasser ${ }^{2}$ ) $71 \cdot 10^{-12}$

$$
\begin{aligned}
& \text { "Regenwasser }{ }^{2} \text { ) . . . . . . . . } 19 \cdot 10^{-10} \\
& \text { " } 0,1 \% \text { ige } \text { Kochsalzlösung }{ }^{3} \text { ) . . . . } 17 \cdot 10^{-8} \\
& \text { " } 0,6 n \quad n \quad 3) . . .91 \cdot 10^{-8}
\end{aligned}
$$

1) Dieses Archiv Bd. 57 S. 394 f. 1894.

2) S. Wi edemann, Elektricität. 3. Auf. Bd. 1 S. 569.

3) Interpolitt nach Landolt a. Börnstein, phys.-chym. Tabellen. 2. Auff. S. 481 . 
Hieraus berechnen sich folgende ungefähre Dichtenbeträge:

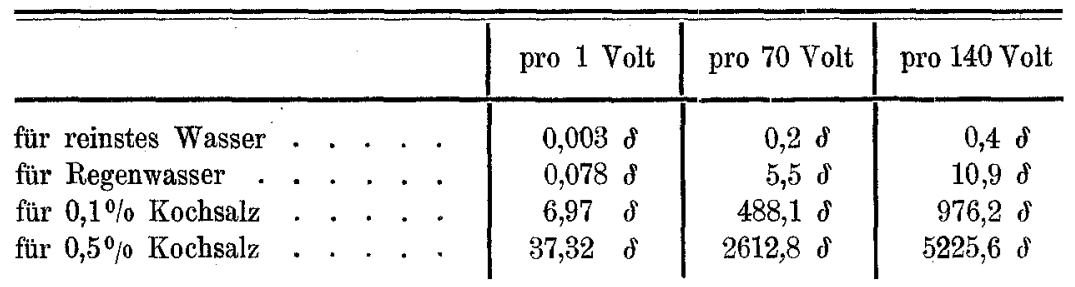

Die Zahlen für reinstes Wasser können für unsere Versuche mit destillirtem Wasser absolut nicht in Betracht kommen, da sie sich auf ein sehr mühsam von Fr. Kohlrausch hergestelltes und sich nicht lange haltendes Product beziehen. In unsern Versuchen, in welchen das destillirte Wasser mit thierischen Theilen in Berührung ist, wird sein Leitungsvermögen eher dem des Regenwassers nahe kommen, die Dichte also zu mindestens $5-10 \delta$ zu veranschlagen sein; im Nerven wird aber, da er die Stromfäden wegen seines besseren Leitungsvermögens in sich zusammenzieht, eine noch erheblich höhere Dichte herrschen. Aus den Versuchen mit 0,1 procentiger Kochsalzlösung darf man schliessen, dass die zum Zustandekommen der Erscheinung erforderliche Dichte im Nerven selbst von der Ordnung mehrerer Hunderte von $\delta$ ist. Zum Vergleich sei erwähnt, dass an in Wasser versenkten Nerven eine Gesammtdichte von $0,05 \delta$ bereits genügt, um die starke Phase des Zuckungsgesetzes zu geben, und dass curarisirte Muskeln in Wasser bei $0,08 \delta$ die ersten Schliessungszuckungen zeigen ${ }^{1}$ ). Jedenfalls dürfen also die zur Hervorrufung des Austritts erforderlichen Stromdichten als relativ ungemein hohe bezeichnet werden.

Die Beobachtung geschah fast durchweg mit Objectiv $\mathrm{C}$ und Ocular 2 von Zeiss, bei Abbe'schem Condensator und stark verengter Irisblende.

Bei der jetzigen Anordnung erlangt nun, wie beabsichtigt war, der Inhaltsaustritt aus dem anodischen Nervenquerschnitt sehr viel grössere Entwicklung als vorher, weil die „Einrollung" sich, wenn überhaupt, erst nach sehr langer Zeit einstellt. Die Länge der pilzförmigen Austrittsfigur, vom Querschnitt aus in der Achsenrichtung gemessen, kann über 30 Faserbreiten erreichen; der Austritt ist makroskopisch leicht sichtbar. Am besten ist es für Nerven, nach

1) Vgl. a. a. O. S. 395 Anm. 
Weiteres üb. d. Wirk. starker Ströme a. d. Querschnitt d. Nerven u. Muskeln. 517

wie vor Wasser zu nehmen, weil in 0,1\%oiger Salzlösung Einrollung 11. s. w. ziemlich früh eintritt.

Ausser dem Austritt und dessen Zurückzucken beim Oeffnen sieht man in Wasser sehr deutlich die blassen Bindegewebsumhüllungen am Längsschnitt des dünnen Nervenstücks am Anodenende anschwellen und bei der Oeffnung zuckungsschnell wieder abschwellen. Ferner erkennt man eine Divergenz der Nervenfasern in der Nähe des anodischen Querschnittes und eine gegenseitige Wiederannäherung beim Oeffnen. Alle genannten Oeffnungswirkungen sind verstärkt beim Schliessen in entgegengesetzter Richtung.

Am Muskel hat die jetzige Anordnung im Vergleich zur früheren weit mehr Neues zu Tage gefördert als am Nerven. Der Muskel leitet beträchtlich besser als der Nerv, wie längst bekannt ist und sich dadurch bestätigt, dass er die Austrittserscheinung auch in $0,6 \%$ iger Kochsalzlösung zeigt. Indess ist vom Gebrauch derselben wegen starker Gasentwicklung und Trübung durch elektrolytische Producte abzurathen. Dagegen ist hier der Gebrauch der $0,1 \%$ igen Kochsalzlösung am Platze, und empfehlenswerther als der des destillirten Wassers. Die für den Versuch nöthigen möglichst dümnen, glatten und mit scharfen Querschnitten versehenen Faserbündel entnimmt man am besten den tieferen Muskeln des Froschoberschenkels, besonders der dem Knochen zugewandten Seite oder dem Innern des Muskels. Man fasst mit einer in feinen Spitzen endigenden Pincette ein möglichst dünnes Bündel, schneidet hinter der Pincette mit der Scheere ein und isolirt nun durch sanften Zug das Bündel von seiner Nachbarschaft; dann schneidet man es so weit von der Pincette wie möglich quer durch, überträgt es sofort auf die trockene Glasplatte, lagert es durch Zug longitudinal, und macht erst jetzt den zweiten Begrenzungsschnitt, indem man das auf der Platte liegende Stück von dem in der Pincette noch gehaltenen Rest abtrennt. Nun wird die vorher auf der Unterfläche mit der Lösung benetzte Deckplatte gleich in richtiger Lage aufgelegt und, falls noch Flüssigkeit zur vollen Erfüllung des capillaren Raumes fehlen sollte, ein Tropfen an den Rand des Deckglases gebracht, und nach erfolgter Vollsaugung des Raumes der Rest mit Fliesspapier entfernt. Das Muskelbündel muss womöglich gleich beim Auflegen auf die Glasplatte die nöthige Dünne haben, weil beim Zerzupfen mit Nadeln die Fasern ihre glatte parallele Streckung verlieren. Wird ein Zerzupfen wegen zu grosser Dicke des Bündels 
nöthig, 'so nimmt man einen Tropfen der 0,1 procentigen Lösung zu Hülfe; die in beschriebener Weise entnommenen, von zwei Querschnitten begrenzten Bündel zerfallen äusserst leicht beim Gebrauch der Nadeln in dünnere, und es ist nur darauf zu achten, diesen eine gerade Lage zu geben, und Berührung der Querschnitte zu vermeiden.

Mit den angegebenen Strömen zeigt nun auch der $\mathrm{Muskel}$ einen ungemein überraschenden Inhaltsaustritt am anodischen Querschnitt, von welchem in der ersten Mittheilung nur Rudimente angegeben sind. Derselbe ist neben dem Nervenaustritt zweifellos die grossartigste mir bekannte mikroskopische Bewegungserscheinung und übertrifft diejenige des Nerven noch an Zierlichkeit. Mit ungemeiner Macht sieht man den Inhalt, und zwar stets sehr ausgesprochen fibrillär gestreift, aus dem Sarkolemm herausquellen, wobei er sich durch Divergiren der Fibrillen verdickt; zugleich aber zieht sich das Sarkolemm lebhaft zurück, indem ein stark lichtbrechender Abschnürungsring längs dem freiwerdenden Inhalt sich rasch zurückstreift. Es ist zweifellos, dass gegenüber der ursprünglichen Fluchtlage des Querschnitts sowohl der Inhalt nach aussen wie das Sarkolemmende nach innen sich bewegt. Der Betrag der Hervorquellung des Inhaltes kann absolut genommen so ziemlich den des Nerveninhalts erreichen; in Muskelfaserbreiten ausgedrückt beträgt er etwa bis zu 3 oder 4 , und ungefähr ebensoviel die Sarkolemmretraction. Schliesslich wird die Erscheinung durch Einrollung unterbrochen.

Sehr bemerkenswerth ist, dass man häufig einzelne Fasern, deren Ende zufällig rückwärts umgebogen ist, sich unter der Einwirkung der Anode, oftenbar unter dem Andrang des fibrillären Inhaltes grade strecken und nun sofort den Inbalt austreten sieht. Diese Gradstreckung kommt aber nur zu Stande, wenn die Faser ziemliche Bewegungsfreiheit hat, d. h. wenn die Flüssigkeitsschicht nicht allzudünn ist. Druck auf das Deckglas ist überhaupt bei allen diesen Versuchen zu vermeiden.

Die Vorgänge am Kathodenende sind weit weniger klar zu entziffern. Oft sieht man lange Zeit gar Nichts, während das Anodenende meist sofort nach der Schliessung den Austritt zeigt. Nach längerer Schliessung werden die kathodischen Faserenden sehr blass und erhalten undeutliche Contouren. Zuweilen sieht es so aus, als ob der fibrilläre. Inhalt sich aus dem liegen bleibenden Sarkolemm 
Weiteres üb. d. Wirk. starker Ströme a. d. Querschnitt d. Nerven u. Muskeln. 519

in die Faser hinein zurückzieht unter Entleerung des letzteren. In einigen Fällen habe ich diese Zurückziehung sehr energiseh sich vollziehen sehen. Beobachtung mit polarisirtem Lichte bestätigte, dass die blassen Sarkolemmenden keinen anisotropen Inhalt hatten.

An frei beweglichen gestreckten Faserenden sieht man nicht selten unter dem Einfluss der Kathode hakenförmige Umbiegung eintreten, so dass der Querschnitt nach der Anode sieht, und nun folgt sofort lebhafter fibrillärer Austritt. Die Kuppe des Hakens entleert sich dabei sichtlich und reducirt sich auf blosses Sarkolemm.

Wenn man dagegen dünne Faserbündel so umknickt, dass beide Hälften dicht zusammenliegen, und nun die Umknickungsstelle, an welcher die Faserlängsschnitte eine convexe Krümmung darbieten, zur Anode macht, so sieht man nur Anschwellung der betr. Faserstellen, die so stark werden kann, dass die Krümmung sich zu einem breiteren Bogen ausrundet; einigemal habe ich hierbei an einzelnen Fasern ein Einreissen des Sarkolemms und hierauf sofortiges fibrilläres Herausströmen beobachtet. Die Kathode wirkt auf solche Umbiegungsstellen blassmachend, ebenfalls unter Anschwellung; deutliche Entleerung nach innen habe ich nur sehr selten constatiren können.

Die Ueberlegung, dass man mit noch höheren Spannungen vielleicht noch mehr sehen kann, und dass sehr kurze Durchströmungen, wenn sie sich nur häufig genug wiederholen, zu denselben Effecten führen müssen wie anhaltende, führte mich auf die Anwendung sehr starker Inductionsströme. Zur Erzeugung derselben wurde ein grosser Ruhmkorff'scher Inductor von $20 \mathrm{~cm}$ Funkenlänge mit Quecksilberunterbrecher verwendet, mit ganz demselben Betrieb wie zur Hervorbringung der Röntgenstrahlen. Hier zählen die Spannungen nach Tausenden von Volt. Schon in der ersten Mittheilung habe ich angeführt, dass ein gewöhnlicher SchlittenInductionsapparat an der Anode der Oeffnungs-Inductionsströme den Nervenaustritt bewirkt. Mit dem grossen Apparat sind nun die Erscheinungen sowohl am Nerven wie am Muskel ungemein grossartig. Der anodische Austritt erfolgt in unzähligen zuckungsartigen Vibrationen, offenbar entsprechend dem Tempo des Unterbrechers. Aber auch am Kathodenende kommen nicht unbeträchtliche Austritte zu Stande, was kein Wunder ist, da hier die Schliessungs-Inductionsströme ihre Anode haben, welche bei so starken Apparaten hinreichend kräftig sind, um ebenfalls zu wirken. Principiell Neues 
aber ergaben die Versuche mit Inductionsströmen nicht. In vielen Fällen werden sie ausserdem sehr gestört und unterbrochen durch starke Erhitzung der durchströmten Flüssigkeit. Man sieht dann plötzlich, namentlich am Muskel, das Präparat durch Coagulation sich in einen verkürzten und verdickten Strang verwandeln, oft auch die Frontlinse des Objectivs sich durch Wasserverdampfung beschlagen ${ }^{1}$ ), da die Deckplatte sehr schmal ist.

Dem Ziele, die hier sich darbietenden Erscheinungen zu begreifen, sind wir, wie mir scheint, etwas näher gekommen. Zweifellos handelt es sich um physikalische Vorgänge im engeren Sinne, d. h. um solche, welche auch am todten Objecte sich abspielen. Auch die Lebenserscheinungen sind sicher in letzter Instanz physikalische, aber von solcher Complicirtheit, dass unsere Erwartung, den Zusammenhang zu durchschauen, sehr herabgestimmt ist, und viele Enttäuschungen erlebt.

Die Erscheinung, um welche es sich hier handelt, ist an die Structur des Nerven und des Muskels gebunden; an keinem anderen Object habe ich bisher etwas Vergleichbares auffinden können. Das Wesentliche lässt sich kurz so ausdrücken, dass der Strom den Inhalt der Röhren dieser Gebilde nach der Anode, die Scheide nach der Kathode drängt. Nebenher spielt sich die gewöhnliche Wasserfortführung im feuchten Gewebe nach der Kathode hin $a b$.

Dass es sich hier nicht um das blosse Reuss-Jürgensen'sche Phänomen handelt, d. h. um die Fortführung suspendirter kleiner Partikel zur Anode, ist ohne Weiteres klar. Trotzdem gehört anscheinend der Vorgang in das gleiche physikalische Gebiet. Die jetzt herrschende, von G. Quincke und dann von Helmholtz mathematisch durchgeführte Theorie dieses Gebietes ${ }^{2}$ ) lässt sich folgendermaassen kurz formuliren. Flüssigkeiten, welche in Röhren irgend welcher Art eingeschlossen sind, besitzen gegen die Röhrensubstanz im Allgemeinen eine Potentialdifferenz, so dass längs ihrer Oberfläche eine elektrische Doppelschicht ausgebreitet ist. Wird nun ein Strom durch die Flüssigkeit in axialer Richtung geleitet, so be-

1) Dieselbe Ursache hatte auch die in $\mathrm{Bd} .67 \mathrm{~S} .261$ angegebene analoge Erscheinung (ohne Deckglas), welche ìch mir damals nicht recht erklären konnte.

2) Die Literatur desselben findet man zusammengestellt in Wie demann's Elektricitätslehre. 3. Aufl. Bd. 2 S. $153 \mathrm{ff}$., $166 \mathrm{ff}$., und in Winkelmann's Handbuch der Physik. Bd. 3 Abth. 1 S. 493 ff. Von späteren Arbeiten sei noch angeführt: Saxén, Ann. d. Physik. N. F. Bd. 47 S. 46.1892. 
Weiteres üb. d. Wirk. starker Ströme a. d. Querschnitt d. Nerren u. Muskeln. 521

wegt sich letztere, wenn das Rohr eng genug ist, durch die elektrische Ladung und die innere Reibung nach demjenigen Pole, welcher entgegengesetztes Vorzeichen hat wie die innere der Doppelflächen; zugleich hat die Wand selbst eine Tendenz zu entgegengesetzter Verschiebung, welche aber nur an frei beweglichen Wandtheilen, oder an den mit solchen vergleichbaren suspendirten Körpern zur Wirkung gelangt, sobald letztere sich elektrisch dem Sinne nach wie die Wand verhalten. Wird umgekehrt der Röhreninhalt durch irgend eine Kraft in axiale Bewegung versetzt, so wird durch Transmission geladener Wandelemente eine elektromotorische Kraft in der Flüssigkeit erzeugt, deren Richtung nothwendig dieselbe ist, wie die der Strömung, welche èin der Flüssigkeitsverschiebung gleichgerichteter galvanischer Strom erzeugen würde ( $Q$ u incke's Diaphragmaströme).

Wendet man diese Theorie auf unsre Erscheinung an, so würde sich ergeben, dass der Röhreninhalt der Nerven- und Muskelfasern sich negativ elektrisch gegen die Scbeide verhält, da er sich nach dem positiven Pol hin bewegt. Ausserdem müsste, wenn es gelänge den Inhalt in den Röhren mechanisch axial zu verschieben, ein galvanischer Strom, resp. eine elektromotorische Kraft entstehen, welche der Verschiebung entgegengesetzt gerichtet ist. Leider ist vor der Hand keine Aussicht, einen solchen Diaphragmastrom der (Nerven und Muskeln nachzuweisen ${ }^{1}$ ).

Die Annahme einer Potentialdifferenz zwischen Inhalt und Hülle der Röhren im angegebenen Sinne genügt jedenfalls, die Haupterscheinung zu erklären. Die Verschiebung des Inhaltes in den Röhren stösst zweifellos auf enorme Reibungswiderstände, und es ist kein Wunder, dass sie nur in der Nähe offener Enden, d. h. am Querschnitte ausgiebig zur Wirkung kommt. Uebrigens sieht man gelegentlich die nach der Anode gerichtete Inhaltsverschiebung sehr deutlich sowohl in Nerven- wie in Muskelfasern, wie für erstere schon in der früheren Mittheilung erwähnt ist (S. 253). Auch in Muskelfasern habe ich Andeutungen davon oft gesehen; ebenso aber ist der Drang nach der Anode an dem Anschwellen der Umbiegestellen und vor Allem an der Gradstreckung gekrümmter Faserenden äusserst deutlich zu sehen. Die Divergenz der ausgetretenen Massen in

1) Erwähnt sei, dass Du Bois-Reymond die Möglichkeit erwogen hat, den Muskel- und Nervenstrom durch Diaphragmaströme zu erklären (Arch. f. Anat. u. Physiol. 1867 S. 492 ; Ges. Abhandlungen Bd. 2 S. 293). Dieser Gedanke hat übrigens nichts zu thun mit dem hier, weiter unten, erörterten. 
Gestalt der mehrfach beschriebenen Figuren könnte einestheils aus gegenseitiger Abstossung derselben durch gleichnamige Ladung hergeleitet werden. Möglicherweise aber genügt zur Erklärung schon einfach der Verlauf der Strömungslinien, welchen die Massen folgen müssen: da für das Zustandekommen der Erscheinung vielleicht wesentlich ist, dass das eingetauchte Object besseres Leitungsvermögen (richtiger Längsleitungsvermögen, im Hinblick auf die von mir gefundenen grossen Differenzen der Längis- und Querleitung) habe als die umgebende Flüssigkeit, so werden in letzterer die Strömungslinien gegen die Faserquerschnitte hin sich zusammenziehen müssen.

Aber da die Faserhüllen nicht starr sind, so werden auch sie, und zwar nach der Kathode bin, sich etwas verschieben müssen. Dass man diese Zurückstreifung an den Sarkolemmen sehr deutlich sieht, ist schon erwähnt. An den Nerven ist hiervon nichts Deutliches zu constatiren; es liegt aber auf der Hand, dass die Neurilemme vermöge ihrer engen Röhrenform sich viel steifer verhalten müssen als die Sarkolemme. Im Gegentheil sieht man am Nerven, wie früher erwähnt, die Neurilemme am Anodenende etwas aus der gemeinsamen Perineuriumscheide heraustreten, was ich dahin ausdrüekte (S. 256 der ersten Mittheilung), dass sie von dem herausdrängenden Mark eine Strecke weit mitgenommen werden. Auch hierfür gibt es ein sprechendes längst bekanntes Analogon. Wenn eine in Glasröhren eingeschlossene Flüssigkeit suspendirte Theilchen enthält, so bewegen sie sich in der Nähe der Wand nach der Anode, in der rasch nach der Kathode strömenden Achsenschicht werden sie dagegen mit nach der Kathode gerissen. Mechanische Adhäsion kann also unter Umständen die elektrische Bewegung umkehren.

Bis hierher handelte es sich nur um Röhrenwand und Röhreninhalt als Ganzes. Mehrfach ist hervorgehoben worden, dass daneben sich die gewöhnliche Elektrotransfusion des Wassers im Gewebe abspielt. Dies ist keineswegs ein Widerspruch. Das Wasser, welches in den feinen Fugen und Poren jedes feuchten Gewebes enthalten ist, verhält sich anscheinend gegen seine Begrenzungen positiv wie in Glasröhren, in feuchtem Thon ete., und wird nach der Kathode geführt. Die Sarkolemme, Neurilemme, Perimysien, Perineurien sind als solche durchtränkten porösen Substanzen anzusehen, ebenso aber auch der Röhreninbalt, welcher weder im Nerven noch im Muskel als einfache homogene Flüssigkeit angesehen werden kann. Alle diese Theile werden also in sich die gewöhnliche Elektrotrans- 
Weiteres üb. d. Wirk. starker Ströme a. d. Querschnitt d. Nerven u. Muskeln. 523

fusion zeigen; auch wäre es kein Wunder, wenn sich in ihnen Körnchenbewegungen nach der Anode fänden. Mit anderen Worten: die specifische Erscheinung, welche uns beschäftigt, ist die gröbere, durch das Verhalten des Inhalts zur Scheide bedingte; die Elektrotransfusion ist der allgemeinere und feinere Vorgang.

Wir haben zur Erklärung unseres Phänomens an der Hand der herrschenden physikalischen Theorie die Annahme gemacht, dass der Inbalt der Muskel- und Nervenröhren sich negativ gegen die Hüllen verhält. Dies müsste sowohl während des Lebens als nach dem Absterben der Fall sein. Wäre es nur für den Lebenszustand gültig, so könnte man versucht sein, den ruhenden Muskel- und Nervenstrom als eine Bestätigung dieser Annahme anzusehen, oder umgekehrt eine Theorie dieser Ströme auf dieselbe Annahme zu gründen. Denn wenn wir einen Querschnitt anlegen, würde der negative Inhalt blossgelegt. Die Grösse der elektromotorischen Kraft der Muskeln und Nerven würde in keinem wesentlichen Widerspruch stehen zu der Ordnungszahl der Potentialdifferenzen, welche zur Erklärung der angeführten physikalischen Erscheinungen theils angenommen werden müssen, theils gefunden sind.

Zwar hat schon du Bo is-Reymond die Möglichkeit discutirt, den Muskel- (und Nerven-)strom auf eine Potentialdifferenz zwischen Inhalt und Hülle im angegebenen Sinne zurückzuführen ${ }^{1}$ ). Er hat bekanntlich diese Möglichkeit verworfen, weil ein querdurchschnittener Muskel auch dann noch den Muskelstrom gibt, wenn man nicht direct vom Querschnitt ableitet, sondern vom Längsschnitt eines zweiten Muskels, der mit seinem Längsschnitt dem Querschnitt des ersteren anliegt. Der Inhalt des ersten Muskels ist, meint er, jetzt an beiden Ableitungsstellen von Hüllensubstanz begrenzt, könnte also keinen Strom zeigen. Diese Beweisführung du Bois-Reymond's, welche als classisch gilt, ist, wie nähere Ueberlegung sofort zeigt, durchaus nicht zwingend, und es ist nur durch den Umstand, dass er dem unversehrten Muskel irrthümlich Strom zuschrieb und daher von vornherein gegen jene Erklärung des Muskelstromes eingenommen sein musste, begreiflich, dass er diese Beweisfübrung für ausreichend halten konnte. Denn es ist klar, dass die Suhstanz, welche am Querschnitt zu Tage tritt, himmelweit verschieden ist von derjenigen, lebendigen, welche mit dem Sarkolemm in Berührung

1) du Bo is - Reymond, Untersuchungen üb. thier. Elektr. Bd. $1 \mathrm{~S} .558 \mathrm{f}$. 
524 L. Hermann: Weiteres üb. d. Wirkung starker Ströme a. d. Querschnitt etc.

ist. Besässe also wirklich der Muskelinhalt eine Negativität gegen das. Sarkolemm, so brauchte sie nicht dadurch aufgehoben zu werden, dass man auch vom Querschnitt durch Anlegung von Sarkolemm, d. h. von dünnen Faserbündeln in unwirksamer Anordnung, ableitet.

Die Möglichkeit, den ruhenden Muskel- und Nervenstrom von einer Negativität des Faserinhalts gegen die Hülle abzuleiten, wäre in der That sehr beachtenswerth, zumal sie die von mir gegen du Bois-Reymond nachgewiesene Stromlosigkeit der unversehrten ruhenden Organe ohne Weiteres ergeben würde, und verdient eine Prüfung, nachdem wir auf ganz anderem Wege zu dieser Annahme gelangt sind. Allein, wie schon angedeutet, muss sie deswegen verworfen werden, weil die Erscheinung, welche uns auf sie geführt hat, ebenso kräftig an todten wie an lebenden Organen auftritt, während doch Muskel- und Nervenstrom nach dem Tode bis auf zweifelhafte Spuren verschwinden. (Auch würde zur Erk]ärung der Actionsströme die weitere, ganz unvermittelt dastehende Annahme nöthig sein, dass erregter Inhalt weniger oder gar nicht gegen die. Hülle wirke.) Die Stromlosigkeit todter Organe, welche quer durchschnitten sind, nöthigt uns anzunehmen, dass der Gegensatz zwischen Inhalt und Hülle, welcher nach der Theorie die Austrittserscheinung verursacht, zu schwach ist, um einen Längs-Querschnittsstrom zu geben, und doch stark genug, um die Erscheinung zu verursachen. Also scheint die Stromlosigkeit der todten querdurchschnittenen Organe mit unserer Annahme wohl vereinbar, der Muskel- und Nervenstrom aber kann nicht auf dieselbe zurückgeführt, sondern muss nach wie vor von dem elektromotorischen Vorgange an der Demarcationsfläche hergeleitet werden.

Nachträglicher Zusatz:

In einer soeben erschienenen Mittheilung von F. Braun (Ann. der Physik. N. F. Bd. 63 S. 324) werden Versuche an Glasröhren und Membronen angegeben, welche zu dem von mir gefundenen Austrittsphänomen in Beziehung stehen sollen. Da mir dieser behauptete Zusammenhang nicht klar geworden ist, so muss ich abwarten, ob vielleicht der Verf. ausführlicher auf denselben zurückkommen wird. 